\title{
Towards Performing Ultrasound-Guided Needle Biopsies from within a Head-Mounted Display
}

\author{
Henry Fuchs, Andrei State, Etta D. Pisano MD*, \\ William F. Garrett, Gentaro Hirota, Mark Livingston, Mary C. Whitton, \\ Stephen M. Pizer \\ Department of Computer Science \\ * Department of Radiology, UNC School of Medicine \\ and Member, UNC-Lineberger Comprehensive Cancer Center \\ University of North Carolina at Chapel Hill \\ Chapel Hill, North Carolina, 27599
}

\begin{abstract}
Augmented reality is applied to ultrasound-guided needle biopsy of the human breast. In a tracked stereoscopic head-mounted display, a physician sees the ultrasound imagery "emanating" from the transducer, properly registered with the patient and the biopsy needle. A physician has successfully used the system to guide a needle into a synthetic tumor within a breast phantom and examine a human patient in preparation for a cyst aspiration.
\end{abstract}

\section{Introduction}

In recent years, ultrasound-guided needle biopsy of breast lesions has been used for diagnosis and has replaced open surgical intervention. Ultrasound guidance is also often used for needle localization of some lesions prior to biopsy, as well as for cyst aspiration. However, ultrasound guidance for such interventions is difficult to learn and perform. A physician needs good hand-eye coordination and three-dimensional visualization skills to guide the biopsy needle to the target tissue area with the aid of ultrasound imagery; typically, the ultrasound data is viewed separately from the patient, displayed on a conventional video monitor. We believe that the use of augmented reality (AR) technology can significantly simplify both learning and performing ultrasound-guided interventions.

AR combines computer-synthesized images with the observer's view of her "real world" surroundings [Bajura 92]. In our application, the synthetic imagery consists of computer-processed echography data, acquired through ultrasound imaging, and geometric representations of the ultrasound probe and of the patient's skin surface. The real-world surroundings are the physician's view of the patient, acquired by miniature video cameras mounted on the physician's head. Tracking systems acquire position and geometry information for patient and physician. A high-performance graphics computer generates the combined imagery in real time. The composite images are presented to the physician user via a video-see-through head-mounted display (abbreviated as HMD in the following). The physician sees the ultrasound imagery "in place," registered with the patient.

With conventional methods, the physician has only a two-dimensional ultrasound image to aid her in the inherently three-dimensional task of guiding a needle to a biopsy target. We hope that our system, by presenting echography data registered 
within the patient, will make these widely-practiced procedures easier to perform, both in the breast and eventually also in other, less accessible parts of the body.

In the following sections we discuss medical issues and motivation and give a brief description of our AR system. We conclude with preliminary experimental results and a discussion of near-term as well as longer-term future work.

\section{Clinical Background}

While screening mammography in conjunction with breast physical examination has been demonstrated to reduce breast cancer mortality, this test generates a tremendous number of breast biopsies [Feig 88, Shapiro 77, Shapiro 82], of which relatively few cases are actually malignant. In fact, among non-palpable lesions that are submitted to needle localization and open surgical biopsy, only $10-30 \%$ prove to be malignant [Schwartz 88, Skinner 88, Landerscaper 82, Wright 86, USPSTF 89]. In recent years, ultrasound-guided percutaneous biopsy (both fine needle aspiration cytology and large bore core biopsy) of non-palpable breast lesions has been used as a supplemental diagnostic test, in large part replacing open surgical intervention for lesions [Fornage 87, Harper 88, Hogg 88]. In addition, ultrasound guidance is frequently used for needle localization of occult lesions prior to surgical biopsy and to drain probable cysts [Kopans 84, Muller 85, Kopans 82, Tabar 81].

Unfortunately, ultrasound guidance for these interventions is not an easy method to learn or perform; it requires extensive hand-eye coordination and a good sense for three-dimensional relationships. During the procedure, the biopsy needle is guided to the target tissue area with the aid of echography images. The data, in the form of realtime, single-slice ultrasound images, is displayed in two dimensions on the sonography unit screen (a conventional video monitor). The operator must learn to alter the position and orientation of the needle with respect to the lesion location in real time. Part of the difficulty in guiding the needle is keeping both the needle and the lesion simultaneously in view, that is, within the ultrasound image slice, as the needle approaches the lesion. Also, both position and orientation of the needle (5 degrees of freedom) must be ascertained, in part, from the two-dimensional ultrasound image. As mentioned before, the ultrasound slice is not registered with the patient; it is viewed separately from the patient. The slice's position and orientation within the patient must in turn be ascertained by the physician using visual and tactile feedback. Establishing correspondence between the biopsy needle and structures within the patient's body is thus an indirect process as far as echography image guidance is concerned. On the one hand, the physician must assess the geometric relationship between needle and ultrasound image, a difficult task since the ultrasound image has non-negligible "thickness." On the other hand, she must assess the geometric relationship between the ("mostly" cross-sectional) ultrasound image and the body of the patient. Precision in both parts of this process is essential for the accuracy of the biopsy procedure but difficult to achieve. With the chest wall, pleura and pericardium in close proximity to the breast, and with lesion size ranging down to only a few millimeters, it is quite important for the needle to be accurately positioned, both in order to avoid complications and to obtain the correct diagnosis [Fornage 87, Gordon 93].

Ultrasound is preferable to stereotactic (mammographic) guidance in some circumstances because it does not use ionizing radiation, the guidance is utilized in "real time" which can significantly shorten the procedure, and there are some parts of 
the breast for which stereotactic guidance is impracticable (namely, in lesions directly against the chest wall and in the superficial subaureolar region) [Parker 93].

\section{Motivation}

We believe that an AR system displaying live ultrasound data in real-time and properly registered to the part of the patient upon which the intervention is performed could be a powerful and intuitive tool. In contrast with the traditional display method for echography imagery, this tool presents a stereoscopic, three-dimensional view of the patient fused with the ultrasound data, which is displayed not only correctly positioned and aligned within the patient, but also at its true scale (life size). (In contrast, in the traditional technique the ultrasound image occupies an entire video display and is viewed at varying degrees of magnification, often larger than life size.) The AR paradigm replaces the indirect two-part process described in Section 2 with a single correlation step, in which the computer-enhanced visual feedback matches the physician's view of patient, needle, and needle entry point, as well as the physician's tactile feedback. We believe the physician performing ultrasound-guided needle biopsy will benefit from such an integrated, unified sensory environment, which we postulate will require significantly reduced "intuition" on the physician's part. We anticipate the following specific benefits for such biopsy procedures:

- Reduced average time for the procedure (benefits both physician and patient)

- Reduced training time for physicians learning to perform needle biopsies

- Greater accuracy in sampling of and distinguishing between multiple targets

- Reduced trauma to the patient through shorter and more accurate procedures

- Wider availability of the procedure due to ease of performing it.

We selected breast biopsy as the first application of this new image display technology for several reasons. First, the breast is relatively accessible since it is located on the outside of the body. This simplifies the logistics of the procedure itself for this pilot developmental phase. Second, the patients are generally healthy and able to cooperate with the use of the new equipment. Third, biopsies can be performed outside a hospital setting (i.e., in the computer science department) without undue risk. The breast contains no major blood vessels or vital organs that might become accidentally damaged if the equipment were to unexpectedly malfunction.

We also chose breast biopsy because it presents realistic clinical challenges. Patient motion has a more pronounced effect on the location of an abnormality than it would with deeper organs. In addition, the lesions within the breast vary significantly in size and location but tend to be smaller than those sampled in deeper locations.

In summary, breast biopsy is procedurally convenient but poses realistic technical challenges. Consequently, if AR technology can meet these challenges, we can expect to eventually apply it to other organs in less accessible locations.

\section{Augmented Reality System}

We have designed and implemented a prototype AR system-described in detail in [State 96] - to aid physicians performing ultrasound-guided needle biopsies. It has been used for phantom experiments as well as human subject experiments and consists entirely of commercial components:

- A stereoscopic video-see-through HMD equipped with miniature video cameras, assembled from a Virtual Research VR4 HMD and two Panasonic GP-KS102 color CCD video cameras with Cosmicar $12.5 \mathrm{~mm}$ lenses. 
- A PIE Medical Model 200 ultrasound machine with a $7.5 \mathrm{MHz}$ linear transducer.

- An Ascension Flock of Birds ${ }^{\mathrm{TM}}$ with Extended Range Transmitter tracking system for tracking the observer's head.

- A FARO Technologies Metrecom IND-01 mechanical arm for precise (tethered) tracking of the ultrasound transducer.

- A Silicon Graphics Onyx Reality Engine ${ }^{2 \mathrm{TM}}$ workstation with 4 high-capacity raster managers, a Sirius Video ${ }^{\mathrm{TM}}$ real-time video capture device and a Multi-Channel Output unit. The Sirius is used for both HMD video acquisition and for ultrasound video acquisition.

The prototype system makes heavy use of the high-speed image-based texturing capability available in the graphics workstation. The video capture subsystem acquires HMD camera video and ultrasound video. The display presented to the user resembles the display offered by an earlier on-line volume reconstruction system [State 94, 95], but the images obtained are superior to the older system's. The new system can sustain a frame rate of $10 \mathrm{~Hz}$ for both stereo display update and ultrasound image capture. It provides high-resolution ultrasound slice display and rendering for up to 255 ultrasound slice images at 256-by-256-pixel resolution. Registration errors (due to distortion and lack of precision in the magnetic head-tracker) are corrected dynamically, by videometric tracking of landmarks in the video image. The live video and computer graphics elements are composited digitally, which prevents artifacts introduced by the external chroma key device used in previous systems.

Each ultrasound slice is presented in its correct location and orientation at the moment of acquisition and displayed properly intersecting with other slices. Old slices dim and fade away in time, controlled by a user-definable decay parameter. This "3D radar display," expected to be useful in scanning moving structures such as fetuses or biopsy needles, reflects decreasing knowledge about imaging targets which have not been recently "visited" by the scanner. A modified Binary-Space-Partition (BSP-[Fuchs 80]) tree algorithm handles intersecting slices properly and manages expiration of old slices efficiently [Garrett 96]. A large number of directly rendered ultrasound slices can give the appearance of a volume data set.

The ultrasound probe is tracked by a high-precision, rigidly tethered mechanical arm (visible in Figs. 1, 4). The arm provides sub-millimeter positioning accuracy and thus guarantees registration between individual ultrasound slices.

The system is also capable of acquiring patient geometry for a specific area of the skin surface. This is currently done via a manually assisted "sweep" before the actual scanning. During the subsequent scanning the acquired geometry is used to render a synthetic opening - a virtual "pit"- embedded within the patient. The ultrasound data (consisting of slices) is displayed within this opening (Figs. 2, 3, 4, 5).

\section{Results}

In April 1995, during an ultrasound-guided needle biopsy procedure on a live human subject, the physician (Pisano) did not wear the HMD as the system did not yet have stereo capability. The HMD was worn by a separate observer. The physician used conventional ultrasound display for guidance. During this experiment's preliminary exploration phase, however, the physician did wear the HMD (Fig. 1, left) and was able to observe correct "registration" between tactile feedback and a volumetric display of a cyst presented within the HMD (Fig. 1, right). 

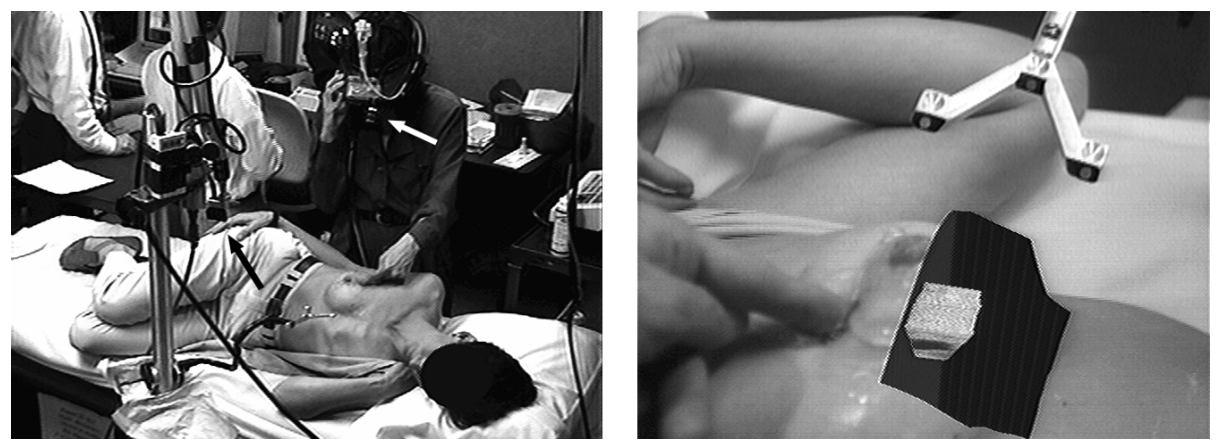

Fig. 1. Left: human subject experiment on April 7, 1995. The physician, wearing a HMD (marked by white arrow) examines the subject's right breast. The black arrow points to the ultrasound transducer attached to the mechanical arm for precise 6 degree-of-freedom tracking. Right: view inside HMD during the experiment in Fig. 1. The synthetic pit contains several ultrasound slices; the frontmost slice is a cross-section through a cyst (dark spot). The physician's finger points to the cyst as she perceives it via tactile feedback. The Y-shaped arm holds landmarks used to maintain correct registration between synthetic imagery and the patient.

In January 1996 the physician, wearing the HMD, successfully guided a biopsy needle into a synthetic tumor within a life-sized breast phantom (Fig. 2). Fig. 3 shows the volume data set acquired by the scanner. It contains images of the cyst and of the needle. The entire procedure was performed using the AR system. In a subsequent human subject experiment, the physician wore the HMD during the exploratory and planning phases preceding the actual biopsy on a human subject, all the way through partial insertion of a cyst aspiration needle. Fig. 4 shows the physician examining the patient and the image she sees inside the HMD.

During these experiments it became clear that the new AR paradigm (or rather, its imperfect implementation in our current system) also has a number of drawbacks:

- The tracked probe is sometimes cumbersome. The accuracy of the mechanical tracker attached to the ultrasound probe (visible in Figs. 1, left, and 4, left) is superior to that of all tracking systems we have experimented with in the past. This accuracy is necessary for correct registration, which is a key element of the AR paradigm. The arm is jointed arm and provides 6 degrees of freedom. Each joint, however, does have a range of motion which is limited to approximately $330^{\circ}$, with hard stops at either end. The physician occasionally was unable to quickly position the probe exactly as she desired due to running into a stop on one of the joints of the arm.

- The physician user must attempt to keep the head tracking landmarks in view at all times. Accurate tracking of the user's head is provided by a hybrid system using a conventional magnetic tracker in combination with vision-based landmark tracking. The techniques require that the landmarks be stationary. The landmarks are mounted on a fixture which is positioned near the patient, but not in the sterile field. (The fixtures are visible all HMD images in this paper) To be effective, the physician has to keep the landmarks in view of the head-mounted cameras, which represents a significant burden. In fact, keeping the landmarks in view proved difficult unless they were positioned so close to the working area as to often physically encumber the physician.

- The pixel resolution of ultrasound slice as it appears within the HMD is poor. Due to the pixel resolution of the HMD, the overall resolution of the images presented to the user is only about $240 \times 200$. In addition, the ultrasound data, as 

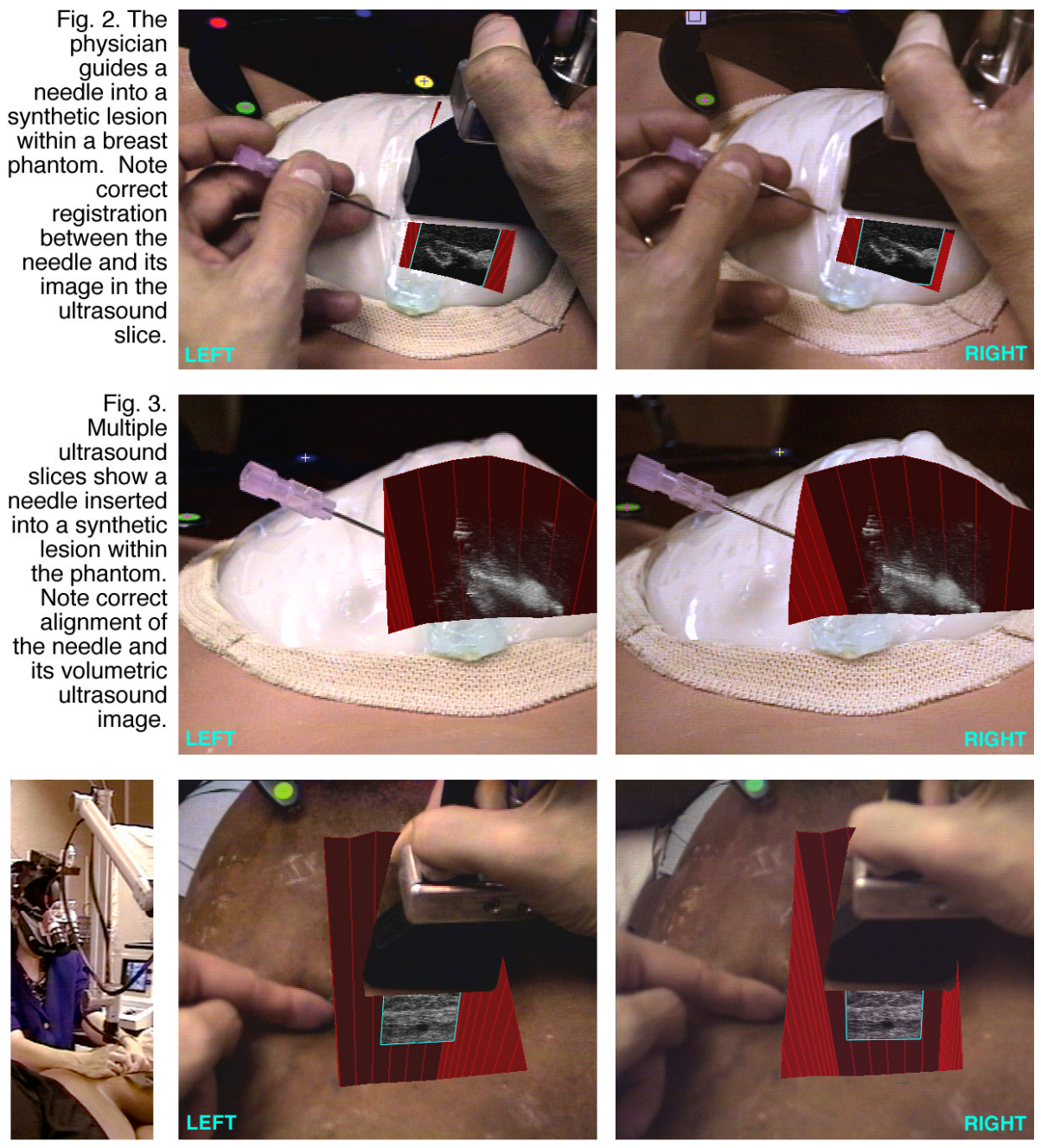

Fig. 4. Left: human subject experiment on January 5, 1996. During preliminary exploration, the physician uses the AR system. The mechanical tracker for the transducer is visible in the foreground

Center and right: stereo view within HMD. The system was not used for the actual intervention.
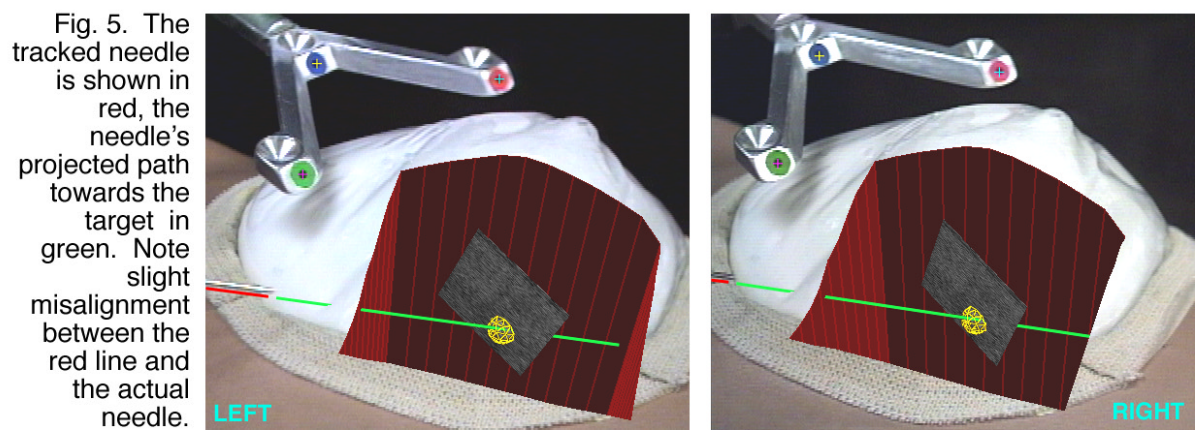
noted above, is only a fraction of the entire viewing area. Together, these characteristics of the system mean that the pixel resolution of the slice is on the order of $40 \times 40 \mathrm{HMD}$ pixels (Fig. 4), compared to the typical video resolution of $500 \times 500$ on the display of a conventional ultrasound machine. Small features within the echography image are not resolved by our system as they are less than one pixel in size. For example, while a 14-gauge biopsy needle was visible within the HMD, a 22-gauge aspiration needle could not be seen in the HMD.

- From the physician user's point of view, the ultrasound slices are sometimes displayed edge-on. At other times, they are occluded by the probe (if the probe is between the physician's head and the ultrasound image). Also, the ultrasound slice occupies only a small portion of the display. These observations are in fact inherent characteristics of the AR paradigm. We believe a simple change of technique is necessary in these situations: the physician must move her head and/or body in order to be able to "see around" visual obstacles or move closer, as one would when a mechanical tool obscured an element that the tool is operating on or when one wanted to have a closer look at a small part. In our current system, the HMD is relatively cumbersome (see above) and other factors such as landmark tracking (see above) also restrict user movement. We therefore expect these visibility problems to become less significant once the usability issues are addressed and the physician will be able to move about unencumbered.

- The video-see-through HMD weighs nearly six pounds, including counterweights for balance, when in use. The unit is heavy, bulky, and somewhat cumbersome. In addition, there is a 4 inch offset between the lenses of the cameras and the user's eyes. While the user can adapt to this offset, it requires a level of training which would better be eliminated from the system.

- Good visual registration of the computer-generated imagery with the patient requires that the patient be still during the whole procedure once we acquire patient geometry data (the patient "sweep"). This data is gathered before the sterile field is created and cannot be re-acquired without compromising sterility. Holding still generally proves uncomfortable for the patients.

- The AR system requires a fairly lengthy set-up process before each patient experiment, on the order of nearly an hour including a series of per-patient calibrations and measurements. While marginally acceptable in this experimental system, clinical use should require easier start-up.

In addition to addressing all the above issues (see next section), it is obvious that our AR paradigm would greatly benefit from the incorporation of a needle tracker into the system. This would allow us to further enhance the display by showing the needle inside the pit and by displaying a projected trajectory of the needle which could be used for "aiming." We therefore conducted a (preliminary) experiment with a tracked needle in a breast phantom and with simple target enhancement techniques (Fig. 5). Since the mechanical tracker is our only high-precision tracker, we had to switch tracking from the ultrasound probe to the needle during the procedure. First, while tracking the probe, we positioned a single ultrasound slice imaging a crosssection through a cyst into the breast phantom. Then we disconnected the mechanical tracker from the ultrasound probe and attached it to the needle. Tracking the needle allows us to display the projected path of the needle during insertion, which we hope will significantly improve guidance. The reason for this is that the physician can aim the needle with the (green) projected trajectory displayed in stereo within the HMD. The (yellow) wireframe sphere also visible in Fig. 5 is an interactively positioned 
target enhancement marker; the needle is aimed at this marker. Since the marker is fixed in space, it can also be used to re-acquire the target after exploration of neighboring tissue with the ultrasound scanner. The marker was initially positioned by aiming at the ultrasound slice with crosshairs displayed within the HMD. This experiment was only marginally successful: despite visibly aiming directly at the target, upon verification with the ultrasound probe after the experiment we determined that we had missed the target in one instance and that we had only tangentially hit the boundary of the target in the second instance. We are currently investigating the sources of these errors, which we speculate may be due to compounded errors from the mechanical tracker (which is used twice, once for the ultrasound slice and once for the needle).

\section{Conclusions and Future Work}

We have implemented an AR system sufficiently robust and accurate that a physician can successfully perform needle insertion into a target inside a standard breast phantom and report that the procedure was easy. We anticipate our physician colleague performing the entire procedure on human subjects using the AR system-i.e., while wearing the HMD - as the system's accuracy and usability are improved in the near future.

Our primary near-term goal is to improve tracking and registration accuracy and incorporate needle tracking. We are currently investigating commercial high-accuracy optical systems for this purpose. We are also on the brink of integrating a new lightweight HMD into the system [Colucci 95]. This unit is characterized by videoresolution displays and a folded optical path for the video cameras. The latter eliminates the eye offset problem. The increased resolution addresses the slice display problem mentioned above.

In order to achieve proper depth relationships between synthetic imagery and the patient's body, the system must acquire and maintain a geometric model of the relevant regions of the skin surface. This is currently done before the actual surgical procedure, in the manually assisted "sweep" phase, during which the skin surface is swept with the mechanical tracker. The drawback of this technique is that it doesn't track skin surface deformations or patient motion. If these occur, the sweep must be repeated. We plan to enhance this component of our system by collaborating with UNC's telepresence research group.

Another goal will be to improve the visualization of intervention targets. The visualization of simple targets such as nodules and cysts is relatively trivial with our current ultrasound slice(s) rendering technique and the simple target enhancement method shown in Figure 5. More complex image analysis and rendering techniques for the ultrasound data will have to be used in order to visualize complex structures or in order to visualize motion of (or detail within) simple structures. We have begun to investigate the applicability of methods such as Whitaker's active blobs technique [Whitaker 94], core-based methods [Pizer 96], and Kalman-filter-based methods. We eventually hope to be able to automatically construct surface models of targets such as cysts from a set of ultrasound slices and then to track their motion and/or deformation from a set of $n$ most recent slices.

In the more distant future, we hope to be able to expand our work to include a new visualization for sampling the abdominal viscera, which are frequently biopsied percutaneously with sonographic guidance using current technology. For example, the application of an advanced version of our AR technology could allow relatively 
easier sampling of lesions within the liver and kidneys. As our methods mature and become considerably more robust, we hope to expand the use of these visualization methods for less accessible organs such as the pancreas and adrenals. Other likely future targets for AR systems are small targets such as foreign body fragments resulting from accident and trauma emergencies. Eventually head-mounted displays and other AR devices may replace conventional displays altogether for some applications.

\section{Acknowledgments}

It takes many people to realize a complex system like the one described here. We wish to express our gratitude to John Airey, Ronald T. Azuma, Michael Bajura, Andrew Brandt, Gary Bishop, David T. Chen, Nancy Chescheir (MD), D'nardo Colucci, Mark Deutchman (MD), Darlene Freedman, Jai Glasgow, Arthur Gregory, Stefan Gottschalk, Ricardo Hahn (MD), David Harrison, Linda A. Houseman, Marco Jacobs, Fred Jordan, Vern Katz (MD), Kurtis Keller, Amy Kreiling, Shankar Krishnan, Dinesh Manocha, Michael North, Ryutarou Ohbuchi, Scott Pritchett, Russell M. Taylor II, Chris Tector, Kathy Tesh, John Thomas, Greg Turk, Peggy Wetzel, Steve Work, the patients, the Geometry Center at the University of Minnesota, PIE Medical Equipment B.V., Silicon Graphics, Inc., and the UNC Medical Image Program Project (NCI P01 CA47982).

We thank the anonymous reviewers for their comments and criticism.

This work was supported in part by ARPA DABT63-93-C-0048 ("Enabling Technologies and Application Demonstrations for Synthetic Environments"). Approved by ARPA for Public Release-Distribution Unlimited. Additional support was provided by the National Science Foundation Science and Technology Center for Computer Graphics and Scientific Visualization (NSF prime contract 8920219).

\section{References}

Bajura M, Fuchs H, Ohbuchi R (1992). "Merging Virtual Objects with the Real World." Proceedings of SIGGRAPH '92 (Chicago, Illinois, July 26-31, 1992). In Computer Graphics, 26, 2 (July 1992), ACM SIGGRAPH, New York, 1992; 203-210.

Colucci D, Chi V (1995). "Computer Glasses: A Compact, Lightweight, and Cost-effective Display for Monocular and Tiled Wide Field-of-View Systems." Proceedings of SPIE; 2537: 61-70.

Feig SA (1988). "Decreased Breast Cancer Mortality through Mammographic Screening: Results of Clinical Trials." Radiology 1988;167: 659-665.

Fornage BD, Faroux MJ, Sumatos A (1987). "Breast masses: ultrasound guided fine-needle aspiration biopsy." Radiology 1987; 162: 409-411.

Fuchs H, Kedem ZM, Naylor BF (1980). "On Visible Surface Generation by A Priori Tree Structures." Proceedings of SIGGRAPH '80; 124-133.

Garrett WF, Fuchs H, State A, Whitton MC (1996). "Real-Time Incremental Visualization of Dynamic Ultrasound Volumes Using Parallel BSP Trees." To appear in Proceedings of IEEE Visualization 1996.

Gordon PB, Goldenberg SL, Chan NHL (1993). "Solid Breast Lesions: Diagnosis with USGuided Fine-Needle Aspiration Biopsy.” Radiology 1993; 189: 573-580.

Harper AP (1988). "Fine needle aspiration biopsy of the breast using ultrasound techniques - superficial localization and direct visualization." Ultrasound Med Biol, suppl. 1, 1988; 14:5-12. 
Hogg JP, Harris KM, Skonick ML (1988). "The role of ultrasound-guided needle aspiration of breast masses." Ultrasound Med Biol, suppl. 1, 1988; 14:13.

Kopans DB, Meyer JE (1982). "Versatile springhook-wire breast lesion localizer." $A J R$ 1982; 138: 586 .

Kopans DB, Meyer JE, Lindfors KK, Buchianieri SS (1984). "Breast sonography to guide cyst aspiration and wire localization of occult solid lesions." $A J R$ 1984; 143: 489.

Lalouche, R.C., Bickmore, D., Tessler, F., Mankovich, H. K., and Kangaraloo, H (1989). "Three-dimensional reconstruction of ultrasound images." SPIE'89, Medical Imaging. SPIE, 1989. 59-66.

Landerscaper J, Gunderson S, Gunderson A, et al. (1982). "Needle localization and Biopsy of Nonpalpable Lesions of the Breast." Surg Gynecol Obstet 1982; 164: 399-403.

Muller JWTh (1985). "Diagnosis of breast cysts with mammography, ultrasound and puncture. A review." Diagn Imag Clin Med 1985; 54:170.

Parker SH, Jobe W, Dennis MA, Stavros AT, Johnson KK, et al. (1993). "US-Guided Automated Large-Core Breast Biopsy." Radiology 1993; 187: 507-511.

Pizer SM, Eberly D, Morse BS, Fritsch DS (1996). "Zoom-Invariant Vision of Figural Shape: The Mathematics of Cores." Submitted to Computer Vision and Image Understanding. Also University of North Carolina at Chapel Hill, Department of Computer Science, Technical Report TR96-004.

Sakas G, Walter S (1995). "Extracting Surfaces from Fuzzy 3D-Ultrasound Data." Computer Graphics: Proceedings of SIGGRAPH '95, (Los Angeles, CA, August 6-11, 1995), Annual Conference Series, 1995, ACM SIGGRAPH; 465-474.

Schwartz G, Feig S, Patchefsky A (1988). "Significance and staging on Nonpalpable Carcinomas of the Breast." Surg Gynecol Obstet 1988; 166: 6-10.

Shapiro S (1977). "Evidence of Screening for Breast Cancer from a Randomized Trial." Cancer 1977; 39: 2772-2782.

Shapiro S, Venet W, Strax P, Venet L, Roeser R (1982). "Ten-to-Fourteen-Year Effect of screening on Breast Cancer Mortality." JNCI 1982; 69: 349-355.

Skinner M, Swain M, Simmon R, et al. (1988). "Nonpalpable Breast lesion at Biopsy." Ann Surg 1988; 208: 203-208.

State A, McAllister J, Neumann U, Chen H, Cullip T, Chen DT, Fuchs H (1995). "Interactive Volume Visualization on a Heterogeneous Message-Passing Multicomputer." Proceedings of the 1995 Symposium on Interactive 3D Graphics. Monterey, CA, April 912, 1995; 69-74, 208.

State A, Livingston M, Garrett WF, Hirota G, Whitton MC, Pisano ED, Fuchs H (1996). "Technologies for Augmented-Reality Systems: Realizing Ultrasound-Guided Needle Biopsies." To appear in Proceedings of SIGGRAPH 96 (New Orleans, Louisiana, August 59, 1996). In Computer Graphics Proceedings, Annual Conference Series, 1996, ACM SIGGRAPH.

Tabar L, Pentek Z, Dean PB (1981). "The diagnostic and therapeutic value of breast cyst puncture and pneumocystography." Radiology 1981; 141: 659.

US Preventive Services Task Force (1989). "Guide to Clinical Preventive Services: An Assessment of the Effectiveness of 169 Interventions." Williams and Wilkins, Baltimore, MD, 1989. Chapter 6, 39-62.

Whitaker, RT (1994). "Volumetric deformable models: active blobs." Proceedings of Visualization in Biomedical Computing 1994, Rochester, Minnesota, October 1994; 122 134.

Wright C (1986). “Breast Cancer Screening.” Surgery 1986; 100(4): 594-598. 